\title{
Activismo y Prácticas Digitales en la Construcción de una Esfera LGTB en España*
}

\author{
Begonya Enguix Grau \\ Universitat Oberta de Catalunya (UOC), Barcelona, Espanha. E-mail: benguix@uoc.edu
}

\section{INTRODUCCIÓN}

$\mathrm{E}$ ste artículo analiza la forma en que la existencia de los colectivos de Lesbianas, Gays, Transexuales y Bisexuales (LGTB), sus prácticas, sus protestas y los medios digitales inciden en la protesta colectiva y en la construcción de una esfera LGTB en España. En particular, se centra en la forma en que los colectivos LGTB españoles utilizan los medios digitales. Si bien trata de entender también cómo otras plataformas digitales se ocupan de cuestiones LGTB y cómo la relación entre recursos digitales, acciones materiales y espacios propios alimenta la idea de la existencia de una comunidad y una esfera LGTB, el foco principal del artículo recae en las prácticas digitales.

Para ello, se cuestiona la utilidad de considerar separadamente y como distintas las actividades digitales y no digitales, ya que sólo mirando de una forma transversal y relacional a través de medios, contextos de

\footnotetext{
* Una versión previa de este artículo fue presentada en la Conferencia de la International Communication Association (Phoenix, Arizona), en mayo de 2012. Agradezco los comentarios realizados por los presidentes de la sección en que se presentó, Adrienne Shaw y Vincent Doyle. Parte de este artículo también fue comentado en un seminario del Grupo de Investigación en Cultura Digital Mediaccions (UOC) en 2012. Las sugerencias de Elisenda Ardèvol, Ana María González Ramos, Edgar Gómez y Beatriz Revelles han sido de gran utilidad para darle forma.
}

DADOS - Revista de Ciências Sociais, Rio de Janeiro, vol. 59, no-3, 2016, pp. 755 a 787. 
acción y prácticas de sociabilidad podemos comprender cómo se construye el sentido de pertenencia a comunidades. Como veremos más adelante, en este caso, las interacciones iniciadas en espacios físicos se imbrican con prácticas digitales y viceversa.

En España, los estudios LGTB son bastante incipientes y hay pocos medios de comunicación tradicionales dirigidos a la población LGTB (revistas, periódicos o fanzines). Consideramos, de este modo, que el activismo LGTB es el principal productor de discurso social legitimado sobre las cuestiones LGTB. Por lo tanto, con el fin de analizar la construcción de una esfera LGTB, se han examinado las páginas web de siete colectivos LGTB en España: dos en Madrid (COGAM, FELGTB $^{1}$ ), dos en Barcelona (Cogailes, FAGC), uno en Andalucía (Girasol), uno en Valencia (Lambda) y una federación de asociaciones (Colegas). Sin embargo, este mapa no estaría completo sin la adición de otros recursos digitales que se han especializado en temas relacionados con lo LGTB: son blogs y páginas web como Cáscara amarga, Universo Gay, En sentido contrario, Ocio gay, Dos manzanas, y AmbienteG. Estas webs son las fuentes más importantes de información digital acerca de los últimos eventos, noticias, o acciones particulares relacionadas con la comunidad LGTB².

Sin embargo, no consideramos que los colectivos activistas LGTB sean, aisladamente, los principales creadores de una esfera LGTB, aunque ocupen un lugar importante por producir los discursos legitimados. La esfera LGTB es una configuración compleja y dinámica que excede el mundo estrictamente activista e incluye, al menos, los siguientes elementos: prácticas digitales (páginas de activistas y otras plataformas y páginas web), grupos sociales (asociaciones activistas y empresariales LGTB, secciones LGTB en partidos políticos y sindicatos y en otras organizaciones no gubernamentales, entre otros), espacios físicos (como la sede de las asociaciones), reuniones, lugares (bares y discotecas comúnmente conocidas como el ambiente) y acciones colectivas y conmemoraciones de las revueltas de Stonewall (Orgullo LGTB) que tienen lugar en más de 30 ciudades españolas, con sus respectivos discursos y prácticas.

La relación entre estos elementos nos servirá para examinar cómo las prácticas digitales son constitutivas de una esfera LGTB, y con el fin de hacerlo, consideraremos distintas acciones de protesta y los medios que se utilizaron para convocarlas. De forma adicional al análisis de 
los sitios seleccionados, se ha realizado un trabajo de campo etnográfico intensivo sobre el activismo LGTB, llevado a cabo durante los últimos tres años, incluyendo entrevistas en profundidad y observación participante. Este trabajo de campo "tradicional" nos ha ayudado a complementar la etnografía digital, identificando los recursos digitales pertinentes y permitiendo un análisis de contenido "denso". Siguiendo los datos obtenidos, hemos establecido dos distinciones analíticas importantes: por un lado, la diferencia entre colectivos activistas radicales, con posicionamientos queer y una fuerte crítica al neoliberalismo capitalista; y asimilacionistas, con posicionamientos cercanos a la defensa de los derechos humanos y la igualdad; por otro, la distancia de los recursos digitales que hemos seleccionado respecto a los recursos (y discursos) activistas.

Tras una discusión sobre las nociones de comunidad y esfera pública que serán puestas en relación con el activismo y las prácticas digitales LGTB, el presente artículo se estructura en torno a otros cinco puntos. El primero es una discusión teórica más general sobre las redes sociales, los lugares físicos y el espacio. Le sigue el análisis de las bases locales y digitales del activismo LGTB en España, prestando atención especial al análisis de las páginas web de siete colectivos activistas. Estas páginas no son los únicos recursos digitales LGTB existentes en España, dado que existen algunas páginas web estables dirigidas a la población LGTB que son una importante fuente de información y son comentadas en la siguiente sección. A este análisis le sigue la exposición de dos movilizaciones LGTB (una en Madrid y otra en Barcelona, las dos mayores ciudades de España) para analizar los medios utilizados para convocarlas y el tratamiento mediático recibido, y se concluye con unas consideraciones finales.

\section{COMUNIDADES Y ESFERA PÚBLICA}

El concepto de comunidad ha sido central en las Ciencias Sociales y es particularmente discutido en la Antropología. En el siglo XIX, estaba presente en la distinción de Ferdinand Tönnies entre Gemeinschaft y Gesellschaft; en la distinción de Lewis Henry Morgan entre civitas y societas y también en la distinción de Park y de Wirth entre lo urbano y lo rural. Estaba implícito en la diferenciación entre la solidaridad mecánica y la orgánica de Durkheim, y es el centro de los debates antropológicos que, sobre las comunidades rurales y los campesinos, se vienen dando desde la década de 1950. 
Para Radcliffe-Brown (después de su estancia en Chicago) el concepto de comunidad estaba en deuda con el funcionalismo estructural. La traducción a contextos no "primitivos" del funcionalismo y la perspectiva holística que Malinowski y Radcliffe-Brown identificaron como características de los contextos "primitivos" llevó a Robert Redfield (1956), el eminente teórico sobre las comunidades, a definir las comunidades como grupos holísticos, homogéneos y cerrados que se resisten al cambio. Las comunidades folk de Redfield se basan en las interacciones cara a cara y en la poca diferenciación social, lo que contrasta con los contextos urbanos más abiertos y heterogéneos. Esta concepción de las comunidades como grupos sociales fácilmente identificables y cerrados ha influido en la creencia común de que son agrupaciones distinguibles de individuos similares cuyas características generalmente basadas en criterios identitarios - los unen entre sí al tiempo que los separan de otros.

Julian Steward (1956), por el contrario, consideró que las comunidades no son entidades acabadas, completas o cerradas. A pesar de que son locales, son interdependientes de sistemas socioculturales más complejos compuestos por familias, otras comunidades e instituciones nacionales, entre otros ${ }^{3}$. De manera similar, Eric Wolf, en la década de 1960, consideró las comunidades como incrustadas en largos procesos históricos que crean configuraciones particulares que no están cerradas, sino abiertas, y son permeables, oponiéndose así a la tradición norteamericana de 1950 - bajo influencia de Redfield (1956) - que destacaba la idea de que las comunidades eran locales y tenían unos valores y una cultura particular. Para Wolf (1971), las comunidades son uno de los extremos de las complejas redes de relaciones sociales que se extienden desde la comunidad a la nación (el otro extremo) a través de niveles intermedios. Las comunidades, en el sentido de Wolf, son flexibles y móviles. Son consideradas como interdependientes del contexto desde una perspectiva compleja y transversal. Las comunidades no están aisladas, ni son identificables, holísticas u homogéneas.

Por lo tanto, los conceptos de homogeneidad/heterogeneidad, permeabilidad/impermeabilidad, así como las características de las relaciones dentro de este sistema social y con los sistemas sociales más amplios se convierten en asuntos clave en la definición de las comunidades. Sin embargo, la creencia en una identidad compartida - o una identificación concebida como identidad - es un aspecto fundamental a considerar pues da consistencia a las comunidades y, como en nues- 
tro caso, puede derivar en el activismo o en el emprendimiento de acciones colectivas.

A pesar del cuestionamiento de Wolf de la idea de que las comunidades son homogéneas y cerradas, lo cierto es que la idea de que las comunidades están formadas por un grupo de personas circunscrito y cohesionado que comparte valores, conocimientos e intereses sigue muy anclada a los imaginarios sociales.

En 1993, con Internet todavía empezando, Rheingold acuñó el término "comunidad virtual" (Rheingold, 1993) para referirse a cibergrupos y redes sociales que compartían conectividad y capital social. Rheingold consideró que las comunidades eran grupos de personas que formaban redes de relaciones sociales en torno a discusiones mediadas por un ordenador:

(son) agregados sociales que emergen de la red cuando el número suficiente de personas toma parte... en discusiones públicas el tiempo suficiente, con el sentimiento humano suficiente, para formar redes de relaciones personales en el ciberespacio (Rheingold, 1993:3) ${ }^{4}$.

De acuerdo con Finquelievich (2000), una comunidad virtual es una "red social con base en el ordenador". Y Komito considera, con Wolf, que:

una comunidad no está fijada en forma o función, es un saco mixto de opciones posibles cuyos significados y concreción son continuamente negociados por los individuos en el contexto de restricciones externas cambiantes. Esto es cierto tanto si los miembros del grupo interaccionan electrónicamente, cara a cara o de ambos modos (Kozinets 2010:9) 5 .

La noción de comunidad también es fundamental para la definición de Habermas de la esfera pública. En 1962 Habermas escribió The Structural Transformation of the Public Sphere y definió la esfera pública como una comunidad virtual o imaginaria que no necesariamente existe en un espacio identificable. En su forma ideal, la esfera pública está "constituida por personas privadas y unidas como un público que articula las necesidades de la sociedad con el Estado" (1989:176). A través de los actos de reunión y el diálogo, la esfera pública genera opiniones y actitudes que sirven para afirmar o desafiar (y guiar) los asuntos de Estado. En términos ideales, la esfera pública es el origen de la opinión pública necesaria para legitimar la autoridad en toda democracia que funcione (Rutherford en Soules, 2008). 
El vínculo entre opinión, público, esfera pública y comunidad, fundamentado en un conocimiento compartido, nos proporciona la base para la construcción de las identidades colectivas y allana el camino para el activismo. Los grupos sociales se estructuran alrededor de las afinidades y las redes sociales que se derivan de tales proximidades y pueden construir identidades que se activan mediante su visibilidad social. En el caso de la esfera LGTB, el activismo LGTB ha jugado un papel importante en la construcción de la visibilidad social, tanto a través de la acción como del discurso. Esta visibilidad tiene un doble efecto, pues fortalece la noción de disidencia sexual, pero también crea la ilusión de homogeneidad e identificación entre los miembros del grupo, reforzando así la idea de que existe una comunidad. En nuestro caso, sólo algunos miembros de esta comunidad son visibles (en general, los que responden a las convenciones sociales sobre cómo deben ser y los que pueden superar el estigma que supone en algunos contextos su "identificación" como persona LGTB). Sin embargo, la visibilidad y las comunidades autodefinidas no sólo son el resultado de la agencia, sino que también son resultado de procesos de categorización y control social, como Foucault (1984) claramente estableció. La homofobia (o mejor la LGTBfobia) ha sido y es un medio importante de control de lo que se califica en algunos contextos como "desviación" social y es fundamental para la constitución y la visibilización de las personas y comunidades LGTB.

Las comunidades autodefinidas contemporáneas están estrechamente relacionadas con la lógica y las estructuras del capitalismo tardío y comúnmente se estructuran en torno a prácticas de consumo. La mediación de las tecnologías para la construcción contemporánea de diferentes esferas de interacción, que conforman públicos particulares, también responde a lógicas y estructuras capitalistas. En consecuencia, como veremos, muchas de las páginas web especializadas que se analizan aquí obedecen a estas lógicas de consumo y están llenas de anuncios publicitarios. Por el contrario, las páginas web de los colectivos activistas sólo ofrecen asesoramiento y / o información, como también veremos más adelante.

\section{REDES URBANAS, LUGAR Y ESPACIO}

Aunque este artículo se centra en la conformación digital de la esfera LGTB, no debemos olvidar el espacio físico - en forma de colectivos, acciones y lugares (bares y discotecas del ambiente) -, pues éste juega 
un papel importante en la construcción de esa esfera. Las acciones colectivas, los colectivos y el ambiente son fenómenos urbanos que conforman y son conformados por las dinámicas (co)constitutivas de ciudades y sexualidades (Knopp, 1995:149). De hecho, en España, los colectivos LGTB son un fenómeno predominantemente urbano, aunque no todas las ciudades tienen colectivos LGTB, ni todos los colectivos tienen el mismo nivel de actividad. En general, cuanto más grande es la ciudad y mayor el anonimato, mayor es la actividad. Esa es la razón por la que hemos decidido centrar nuestro análisis en los colectivos de las ciudades más grandes de España (Madrid, Barcelona, Valencia y Sevilla), ya que tienen los índices más altos de actividad LGTB y los colectivos con mayor número de militantes.

Muchos autores han puesto de manifiesto ${ }^{6}$ que las redes de sociabilidad homosexual han existido desde hace mucho tiempo. Las ciudades son lugares idóneos para la construcción de muchas culturas gays y lesbianas contemporáneas (Hubbard, 2012). Eso se debe a que las ciudades y las sexualidades "reflejan cómo se organiza, se representa, se percibe y entiende la vida social y cómo distintos grupos se manejan en estas condiciones y reaccionan a ellas" (Knopp, 1995:149).

En una perspectiva inspirada por Lefebvre, las sociabilidades y los activismos LGTB se ponen de manifiesto en la topografía (lugares genéricos de sociabilidad), los eventos (bailes, manifestaciones del Orgullo) y las organizaciones (colectivos). De esta manera, los colectivos LGTB constituyen un espacio que existe en una relación mutuamente constitutiva con las identidades subjetivas (van Ingen, 2003).

Partiendo de la conceptualización sobre el lugar y el espacio propuesta por De Certeau (1984:117), consideraremos que los lugares son configuraciones instantáneas de posiciones que indican una cierta estabilidad mientras que el espacio se compone de intersecciones de elementos móviles. Esta noción de espacio entronca a la perfección con nuestra consideración de una esfera LGTB. En nuestro caso, los lugares están ocupados por la sede de los colectivos mientras que el espacio LGTB (o, en la terminología de Habermas, la esfera LGTB) se construye gracias a la combinación del lugar (un contexto específico) y el conjunto de actividades digitales o de cualquier naturaleza que se organizan en torno a cuestiones LGTB y que ayudan a conformar las relaciones sociales en las que se negocian poder y significado. Como explica Skeggs, 
la conversión del lugar en espacio interviene para producir una "autenticidad" que posibilita que se produzcan y se reaccione a las demandas políticas. El espacio no es sólo un trasfondo pasivo del comportamiento humano sino que es constantemente producido y reconstruido a medida que los grupos luchan por el poder (o contra la falta de poder) para convertir su "realidad" en conocimiento público, su presencia visible en reconocible (Skeggs et al., 2004:1839) ${ }^{7}$.

El espacio interviene activamente en la definición de la opinión pública, contribuyendo a la esfera pública, y también produce identidades sexuales, visibilidad, comunidades y lugares de resistencia. Un buen ejemplo de estos procesos es el uso del espacio para la protesta social. Por ejemplo, en los años 1970 y 1980, los gobiernos locales comenzaron a conceder permisos para eventos como las marchas del Orgullo (Forsyth, 2001), transformando y sexualizando el espacio público.

De hecho, la ocupación de los espacios públicos urbanos puede ser un medio poderoso de desestabilizar y socavar los procesos de opresión LGTBfóbica para lesbianas, gays, transexuales y bisexuales. Esa ocupación implica tácticas políticas como marchas, performances, manifestaciones del orgullo LGTB y kiss-ins o "besadas", besos colectivos que se organizan para llamar la atención sobre los derechos LGTB (Hubbard, 2001:61). Las besadas y otras protestas como las manifestaciones del Orgullo LGTB atraen la atención del público a través de la exposición de lo privado (Cruces, 1998).

Las redes sociales son básicas para la construcción y la ocupación del espacio público y son constitutivas de las comunidades. Según Chauncey (1994), en los inicios del siglo XX, el aumento de las redes de hombres gay y de la fairy culture (cultura del afeminamiento masculino o cultura del homosexual afeminado) se hicieron más visibles, por lo que las comunidades gay reclamaron espacio público y físico (Taylor, Kaminsky y Dugan, 2002:104). Este proceso produjo espacios queer más o menos permanentes, lugares llamados "barrios", "zonas" o villages. Estas áreas son fundamentales para la reproducción social, económica y cultural de las comunidades de gays y lesbianas (Castells, 1993 en Hubbard, 2001:61). Aunque algunos se muestran escépticos sobre cómo la cultura gay se ha mercantilizado y representado en lugares como West Hollywood, Castro en San Francisco, o Canal Street en Manchester, otros muchos consideran que esos lugares son fundamentales para visibilizar la reivindicación de una plena ciudadanía sexual 
(a pesar del notable sesgo blanco, de clase alta, cosmopolita y de apertura sexual de esos lugares) (Hubbard, 2001:61).

En consecuencia, colectivos, bares, parques o lavabos son elementos físicos de la esfera LGTB. Constituyen y son constituidos por comunidades autodefinidas sobre la base de la disidencia sexual y / o la atracción por el mismo sexo. Estas comunidades habitan el espacio vivido, el terreno de la lucha social, de los contra-discursos y la resistencia a la LGTBfobia y al sexismo (Lefebvre en van Ingen, 2003:202). Una parte de los miembros de la comunidad apoyan el activismo contra la discriminación y la LGTBfobia, y algunos de ellos se convierten en activistas; las relaciones entre la comunidad y el activismo no son evidentes ni estables: las comunidades son siempre mayores que el colectivo de activistas, y, paradójicamente, el activismo - al asentarse en una esfera compartida e intereses particulares - sostiene y al mismo tiempo fragmenta la comunidad (Taylor, Kaminsky e Dugan, 2002).

Activistas y otras personas vinculadas a las comunidades LGTB no sólo generaron y generan un espacio físico, sino que las actividades, acciones, prácticas, y discursos sociales no digitales son ahora completadas, alimentadas y / o controladas y restringidas por prácticas digitales (co)constitutivas de la esfera LGTB.

\section{LA CONSTRUCCIÓN DE LA ESFERA LGTB EN ESPAÑA: ACTIVISMOS ENTRE LO LOCAL Y LO DIGITAL}

En los inicios del activismo LGTB, el activismo y las comunidades estaban muy localizados. Stonewall (1969) y las otras revueltas gay de finales de la década de 1960 (Armstrong, 2002) se llevaron a cabo en lugares específicos y, pronto, se emularon en ciudades de América Latina y de Europa. En España, el eco de Stonewall llevó a la fundación del clandestino Movimiento Español de Liberación Homosexual en Barcelona, en 1970. Este grupo se convirtió en el Front d'Alliberament Gay de Catalunya en 1975 y organizó la primera manifestación por la liberación sexual en España en 1977. En 1980, se legalizaron los "colectivos gay" (como se los denominaba entonces) y, en esa década, florecieron las expresiones de diversidad sexual (antes del azote del SIDA), en zonas como el barrio de Chueca en Madrid (el único espacio territorial que se identifica claramente como LGTB en el Estado) ${ }^{8}$.

Del contexto local, con colectivos y zonas de bares, se pasó al contexto territorial (con colectivos con un mayor radio de acción sobre todo en 
Cataluña, Madrid, Valencia y el País Vasco) y se favoreció el establecimiento de redes mediante la creación de colectivos con una orientación más global: en los años 1980, las asociaciones LGTB españolas se agruparon en la COFLHEE (Coordinadora de Frentes de Liberación Homosexual del Estado Español) y en 1992 se creó la Federación Estatal LGTB (FELGTB). Esta federación representa en España el salto de las asociaciones locales a la política nacional. La FELGTB acogía en 2014 a más de 60 asociaciones de toda España, aunque la distribución geográfica de los grupos no es uniforme: algunas partes de España no tienen ninguna asociación LGTB (en particular las ciudades pequeñas y las zonas rurales), lo que confirma la suposición de Aldrich (2004) acerca de la relación entre la homosexualidad y los entornos urbanos.

También hay asociaciones supranacionales, como la ILGA (Asociación Internacional de Gays y Lesbianas, de la cual FELGTB es miembro), Interpride (la Asociación Internacional de Organizadores del Orgullo) y la EPOA (European Pride Organizers' Association). Las políticas de la identidad se convirtieron rápidamente en "globales" y un entrelazamiento tan precoz de los procesos globales y locales (subrayados y desarrollados mucho más tarde gracias a Internet) podría hacernos pensar en una "glocalidad" temprana (Meyrowitz, 2004).

Para hacer un mapa de la esfera activista, también habría que tener en cuenta las secciones LGTB en los partidos políticos progresistas y en sindicatos. Asimismo, junto con las asociaciones activistas locales, nacionales y supranacionales (nos referimos específicamente a ILGA), es importante igualmente considerar el ambiente (bares y discotecas que a veces colaboran en campañas activistas), la sede de las asociaciones (donde los miembros se reúnen y pueden recibir ayuda y asesoramiento), y las páginas web oficiales de los colectivos activistas. Puesto que muchos activistas se relacionan en el ambiente, estos locales dirigidos al público LGTB son el eslabón entre el activismo LGTB y la población en general, y el tiempo de ocio funciona como un canal de comunicación. De este modo, el ambiente identifica y señala un espacio como LGTB. Además, aparece en muchas páginas web no activistas a través de anuncios: la mayoría de estas páginas son patrocinadas por establecimientos comerciales que se anuncian en la página. De hecho, esta es una de las grandes diferencias que se puede encontrar entre las páginas activistas y las no activistas, pues en las webs activistas no hay publicidad.

En resumen, podemos distinguir diferentes niveles activistas: local (incidencia mayor); nacional (por ejemplo, la FELGTB, cuyo poder de 
interlocución es mayor que el de sus miembros tomados por separado), transnacional y digital. Estos niveles son estables, pero están en permanente flujo: sus relaciones no les preceden ni están constituidos por ellas. Es el discurso sobre cuestiones LGTB lo que les confiere un papel significativo en la configuración de la esfera LGTB y en la política en general, pues este discurso es reconocido por las instituciones políticas del país como la voz de las comunidades LGTB. Por ejemplo, uno de los primeros eventos organizados por el Rey y la Reina de España después de ser coronados en 2014 fue la reunión con diferentes líderes de organizaciones no gubernamentales, entre ellos, el presidente de la FELTGB y de la Fundación Triángulo, dos asociaciones LGTB.

Por tanto, se deposita en algunas asociaciones LGTB - que, a su vez, consideran ser representativas de las realidades LGTB - la responsabilidad social de representar la esfera LGTB. Así, se considera que sus discursos condensan un sistema de valores compartidos por una "comunidad" que comparte también conocimientos, prácticas, experiencias y reivindicaciones.

Pero el activismo español no es una realidad unificada ni única: podemos hablar de un activismo oficialista (asimilacionista), cuyas demandas se basan en los derechos humanos y la igualdad; y de un activismo radical que aspira al cambio social más profundo, considera la liberación sexual como un elemento de la liberación humana y adopta posiciones queer y anticapitalistas ${ }^{9}$. En este artículo, hemos trabajado con colectivos representativos de ambos posicionamientos. COGAM, Lambda y FELGTB representan la perspectiva oficialista, siendo esta última considerada como depositaria de las reivindicaciones por los derechos LGTB. En cambio, la Coordinadora Gay-Lesbiana (que desapareció como grupo en 2014) y el FAGC son representativos de la postura más radical. Girasol ${ }^{10}$ y la Federación Colegas no comparten los puntos de vista más radicales, pero son críticos con el monopolio de las identidades LGTB por parte de la FELTGB y no forman parte de esa federación. Colegas, por su parte, fue relacionada por los líderes LGTB que entrevistamos, con posiciones conservadoras y cercanas al Partido Popular.

\section{Activismo Oficialista y Activismo Radical: Descripción de las Páginas Web de Colectivos y Federaciones}

En su página web oficial, COGAM (www.cogam.es) ${ }^{11}$, una asociación LGTB de Madrid, ofrece información actualizada sobre noticias, pre- 
senta sus servicios (jurídicos, psicológicos y de asesoramiento médico), muestra los diferentes grupos de trabajo (jóvenes, lesbianas, gays, personas mayores, padres y madres, espiritualidad), y organiza actividades de grupo, como excursiones y senderismo. Como objetivos del colectivo, se explicitan los siguientes: apoyo, información, asesoramiento, seguimiento, grupos de trabajo, capacitación, asistencia telefónica, investigación y actividades sociales. En línea con su enfoque asimilacionista, se hace hincapié en la protección de los derechos humanos y de la pluralidad en la sociedad, en la promoción de la igualdad legal/ real y en la lucha contra la homofobia. También ofrece apoyo y asesoramiento a la población VIH. Se promueve la sociabilidad a través de grupos para practicar el senderismo, para ir al teatro, para la conversación en inglés; existe también un canal de radio y un grupo de eurofans ${ }^{12}$ y un blog informativo. En conjunto, el colectivo parece estar centrado en tres funciones: socializar, reivindicar y educar a través de los distintos servicios de orientación (Asesoría; Gay Inform/Linea Lesbos; VIH Prevención) y los diferentes grupos (Stop Homofobia; Derechos Humanos; Senderismo; Radio, etc.). Según los datos proporcionados por su presidente en junio de 2014, COGAM era el mayor colectivo activista de España con unos 300 militantes. A su página web hay que sumar su cuenta de Twitter (9709 seguidores), creada en 2008 y una página en Facebook (7792 likes y 45 visitas) ${ }^{13}$.

La FELGTB (www.felgtb.org), creada en 1992 y con sede en Madrid, se basa en tres principios: la reivindicación de la igualdad jurídica y el respeto a la diversidad afectiva y sexual; la denuncia y reivindicación (celebración del Orgullo Estatal) y la formación de los activistas. Su web se estructura en torno a la información y la documentación sobre temas como la educación, las familias, las políticas lesbianas, jóvenes, etc. Incluye documentos textuales y visuales (videos) sobre diferentes temas y muestra un anuncio que anima a denunciar la LGTBfobia y a colaborar con la FELGTB trabajando en alguna de sus áreas (trabajo, juventud, bisexuales, transexuales, salud, prevención del VIH y otras). Se muestra información exhaustiva sobre distintas noticias como, por ejemplo, la campaña contra los mitos de la bisexualidad o a favor de la salida del armario de lxs jóvenes. También ofrece recursos educativos para la educación en la diversidad y para la orientación a individuos y familias. Puesto que no se trata de un colectivo sino de una federación, no se ofrecen actividades de socialización como se ofrecían en COGAM. El Twitter de la FELGTB se creó en 2010 y es más popular que 
el de COGAM: tiene 17,3 K seguidores y 12,6 K de tuits. Su página de Facebook tenía en el momento del análisis 28622 likes y 70 visitas.

COGAM y FELGTB son los organizadores de la manifestación estatal del Orgullo LGTB, la mayor de Europa con más de un millón de participantes de acuerdo con los datos de la organización.

En Barcelona, la Coordinadora Gai Lesbiana (www.cogailes.org), también una federación de colectivos, no pertenecía a la FELGTB. Inactiva en 2014, hasta 2013 su página web incluía informaciones diversas y ofrecía servicios de asesoramiento jurídico y psicológico o para la detección del $\mathrm{VIH}^{14}$. En su portal destacaban la defensa de los derechos y la lucha contra el VIH. El grupo no se estructuraba sobre grupos de interés, sino en base a dos comisiones, una sobre derechos y otra sobre solidaridad internacional y formación. El contenido de su página se completaba con un anuncio con un número de teléfono gratuito para la defensa de los derechos y alojaba una intranet con un servicio de correo.

También en Barcelona, el Front d'Alliberament Gai de Catalunya (FAGC) (http: / / www.fagc.org /) tiene una página web completamente diferente a las comentadas hasta ahora. El FAGC no es miembro de la FELGTB y es el heredero del colectivo creado en la década de 1980. De-

Figura 1

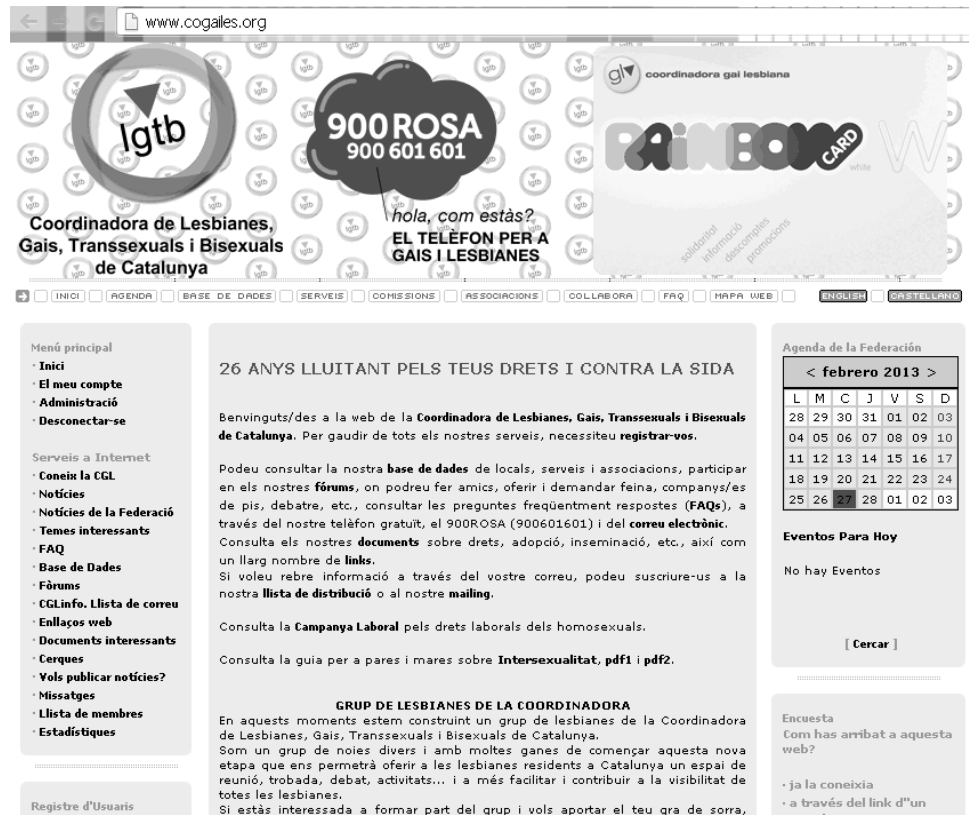


fienden que la liberación sexual debe estar ligada a la liberación social y su espíritu anticapitalista les sitúa como activistas críticos o radicales en contraste con el activismo asimilacionista. Como Eugeni Rodríguez, su cabeza más visible, dijo en una entrevista ${ }^{15}$, hoy no se pueden separar las reivindicaciones LGTB de las reivindicaciones nacionalistas. Su página web es muy simple e incluye direcciones de correo electrónico de los diferentes lugares en los que existe una sección (Barcelona, Girona, Ripollet) y alberga un dispositivo en línea para denunciar las agresiones homófobas: esto está relacionado con el hecho de que el representante del grupo, Eugeni Rodríguez, es también el presidente del Observatorio contra la Homofobia, que cuenta con el apoyo de las instituciones catalanas cuyos logotipos también podemos encontrar en la página web. En la página principal podemos encontrar también un anuncio para convertirse en miembro y colaborar económicamente con la organización. En la sección "documentos y artículos" encontramos posters y anuncios de acciones. El grupo tiene cuenta en Twitter desde 2011 (5.442 tuits y 1.443 seguidores) y su subgrupo en la población de Ripollet (38.000 habitantes) tiene una página en Facebook (desde abril de 2014) con 186 likes.

Al buscar la Coordinadora Girasol (Andalucía), el navegador nos lleva a una página web compartida entre esta federación de grupos y la celebración del Orgullo de Andalucía (http:/ / orgullodelsur.org/girasol). Las últimas noticias son de mayo de 2014 y el objetivo de la página es

Figura 2

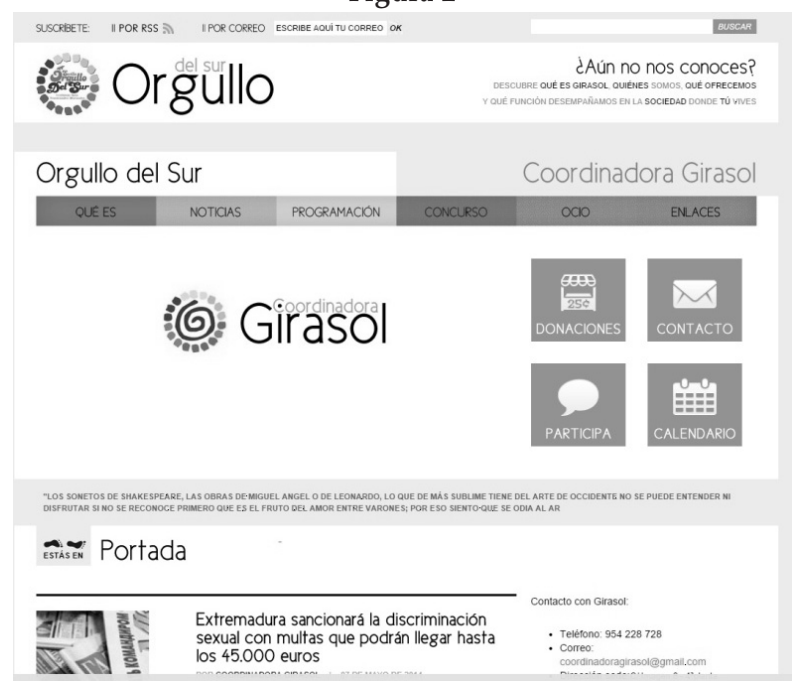


principalmente informativo, incluyendo noticias relacionadas con cuestiones LGTB (la organización de seminarios, por ejemplo) y sobre los eventos del orgullo. Hay una llamada a ponerse en contacto, hacer una donación y participar en las actividades, mediante el envío de un correo electrónico o una llamada telefónica (como en el FAGC).

Su página de Facebook no está activa en octubre de 2014 y su página de Twitter - creada en febrero de 2011 - muestra poca actividad en 2014 (358 tuits y 981 seguidores).

Ya en Valencia, el Col.lectiu Lambda (http://www.lambdavalencia.org/), miembro de la FELGTB, también se estructura en torno a las actividades de diferentes grupos (Espiritualidad, Jóvenes, Derechos Humanos, Lesbianas y Stop Sida). En el momento de la investigación su portal está dedicado a la salud sexual y promete anonimato en la realización de las pruebas del VIH. También ofrece talleres educativos sobre cómo vivir en pareja, información sobre el colectivo y un anuncio para las donaciones, además de información sobre las diferentes reuniones de grupos. Es bastante similar a las webs de los otros grupos pertenecientes a la FELGTB. Su grupo de Facebook data de 2008 y cuenta con 4887 likes. Su cuenta de Twitter fue creada en octubre de 2010 y tiene 3.179 seguidores y 1.927 tuits.

Finalmente, la Federación de Asociaciones Colegas (www.colegaweb.org), con delegaciones en diferentes ciudades españolas, tiene una página web que es principalmente informativa sobre sus acciones: en el momento de la investigación, se agradece al embajador de Estados Unidos su invitación a la celebración de su primer año en España, se exige un cambio en la Constitución española para hacerla más inclusiva y se exige la retirada del obispo de Alcalá por ser un apologista de la intolerancia. También ofrece, como es habitual en todos los grupos analizados, una explicación de quiénes son, un archivo y los medios para ponerse en contacto con ellos. Su cuenta de Twitter tiene 7.155 seguidores y 2.125 tuits, y fue creada en 2009. Su página de Facebook, organizada por la sección de Madrid, tiene 2.003 likes.

\section{Modelos Distintos y Tendencias}

A partir de estas informaciones y descripciones generales se pueden distinguir tres modelos diferentes seguidos por los grupos activistas. El primero es el modelo seguido por COGAM, FELGTB y Lambda, organizados en grupos de trabajo que implican la presencia física de la persona. El segundo modelo es el seguido por Colegas, en el que está 
presente el asesoramiento y la asistencia, así como un servicio de noticias, igual que en el modelo anterior, pero cuya organización interna no se basa en grupos de trabajo. El tercer modelo es heterogéneo e incluye al FAGC (cuya página web es muy simple y centrada en la lucha contra la LGTBfobia), Girasol (que realmente no tiene una página web y se centra en el orgullo) y la Coordinadora Gai Lesbiana (no activa en el 2014 debido a que el grupo no es estable por el momento), que se organiza en torno a dos comisiones, una sobre los derechos y una sobre internacionalización.

A pesar de estas diferencias, y de algunas excepciones, las actividades más importantes de estos colectivos y sus medios de comunicación están profundamente arraigados en el espacio físico y la sociabilidad no digital: las reuniones de grupo, la convocatoria de acciones, los medios de información disponibles apuntan a este hecho. La mayoría de los colectivos ofrecen el teléfono como medio para pedir ayuda y el teléfono y el mail como medios de contacto. Sin embargo, el uso de los medios digitales ha aumentado rápidamente en los últimos años. Todos los grupos consultados tienen una página de Twitter.

En los últimos tiempos (sobre todo después de las revueltas de la primavera árabe, el movimiento 15-M, y las manifestaciones de Occupy Wall Street), el activismo digital o ciberactivismo se ha convertido en una característica clave de los movimientos sociales contemporáneos. Hands (2011:187) considera que el activismo digital promueve la cooperación, la solidaridad y la comunidad, la maximización de la cooperación entre diferentes sectores - desde las fábricas, a los movimientos sociales, a los circuitos de comunicación del movimiento global por la justicia social-, y además se rige por la elección. El activismo digital articula lo local, lo nacional y lo internacional. Sin embargo, como veremos, no debemos confundir los elementos digitales de grupos activistas (como sus páginas web) con la existencia de activismo digital puesto que éste requiere de acciones explícitas y no puede derivarse simplemente de la existencia o la posibilidad de recursos digitales. Además, como hemos visto, las páginas web de los colectivos activistas se centran más en la información, el asesoramiento y las noticias que en la movilización social.

\section{OTROS RECURSOS DIGITALES Y OTROS PÚBLICOS}

Los contextos urbanos favorecen el anonimato, pueden neutralizar el estigma y son el escenario ideal para estructurar prácticas, experien- 
cias y lugares de ocio LGTB que dan forma a comunidades y activismos. Existen hoy otros contextos que también facilitan el anonimato, la neutralización del estigma, la comunicación, o la acción social: se trata de recursos digitales especializados en cuestiones relacionadas con el colectivo LGTB. La construcción de la esfera LGTB no sólo consiste en los establecimientos comerciales y en los colectivos activistas. También está formada por los medios de comunicación LGTB (en España en 2014 solo hay una revista LGTB en papel, Shangay) y por recursos digitales que funcionan como potentes medios de comunicación. Entre estos recursos, destacan:

Páginas web oficiales de los colectivos activistas: como hemos visto, estas páginas ofrecen por lo general información sobre las funciones del colectivo (asistencia, consultoría, organización). Su objetivo es satisfacer las necesidades personales y sobre todo locales, aunque su discurso apunta a demandas globales. Estos colectivos son los depositarios del discurso LGTB legitimado institucionalmente.

Páginas web estables (periodismo digital): las páginas más importantes son: Cáscara Amarga, Universo Gay, En Sentido contrario, Ocio Gay, Dos manzanas, y AmbienteG. Estas páginas son la fuente más importante de información acerca de los últimos eventos, noticias o acciones particulares relacionadas con la "comunidad" LGTB.

Páginas en redes sociales: principalmente Facebook y Twitter.

Páginas de opinión (por ejemplo blogs): entre los blogs dedicados total o parcialmente a la experiencia y/o las reflexiones sobre temas LGTB, encontramos Hijos homosexuales (http://hijoshomosexuales.blogspot.com.es/) y Palabra de artivista (http://blogs.publico.es/shangaylily/), entre muchos otros.

Este trabajo se centra en los dos primeros, en el análisis de las webs activistas en relación con otras webs LGTB especializadas ("webs estables" de ahora en adelante) pues consideramos que las diferencias entre ellas ilustran las líneas maestras de las prácticas digitales en relación con una esfera LGTB. Ambas son las fuentes más importantes de información sobre cuestiones LGTB. Mientras que las webs estables admiten comentarios, mejorando así el "efecto comunidad" de compartir un interés y una opinión (como los blogs), las webs activistas no admiten ningún comentario pero, en cambio, son consideradas como una fuente fiable y bien conocida. Cuando las webs incluyen publici- 
dad, como es el caso de las webs estables, la fiabilidad e imparcialidad de la información ofrecida suele ser cuestionada. Por tanto, la presencia de publicidad en una web es un elemento fundamental para valorar la distancia de esa página respecto al activismo, pues tanto los discursos activistas homonormativos como los discursos activistas críticos entienden la reivindicación como alejada e incluso incompatible con la comercialización ${ }^{16}$. A través de la publicidad, las webs estables se distancian del activismo "puro" y se conectan con espacios de sociabilidad LGTB que sustentan (y fragmentan) comunidades.

Como vemos en los ejemplos que siguen, algunas de las páginas están más cercanas al activismo que otras. Por ejemplo, en Dos manzanas (www.dosmanzanas.com/, Facebook, Twitter y Youtube) el diseño es simple, en colores claros, se destacan las noticias, hay incluso un anuncio para las donaciones (como en las páginas activistas) y poca publicidad. Las noticias se tratan en profundidad y todas tienen una estrecha relación con la realidad LGTB: en el momento de la recuperación ${ }^{17}$, por ejemplo, la primera página se dedicó a la resolución de las Naciones Unidas contra la homofobia y la transfobia.

Ocio gay (http:/ / www.ocioxocio.com/category/ociogay/, Facebook, Twitter, RSS) visualmente hablando también está cercana a las webs activistas dada la centralidad de las noticias y la escasa publicidad que contiene. La mayoría de las noticias están relacionadas con el ámbito LGTB ("Pride en Cáceres", "más de 32\% de los sacerdotes son homosexuales", son algunos de sus títulos), pero también ofrece información sobre música, teatro, salidas, etc. Como en el caso anterior, los colores utilizados son suaves, claros y ponen de relieve el contenido más que una preocupación por el diseño, aunque se hacen algunas concesiones a los colores brillantes.

En Sentido Contrario (www.ensentidocontrario.com) está diseñada como un periódico tradicional y ofrece noticias y publicidad. Combina información sobre la represión en Moscú del orgullo LGTB y el nuevo hit de Britney Spears. Incluye un anuncio para la venta de perfumes, un chat gay y una guía gay. Esta web, por tanto, parece estar destinada exclusivamente a hombres.

En el extremo opuesto, webs como AmbienteG, Universo Gay y Cáscara Amarga están diseñadas con colores brillantes y muestran símbolos de la comunidad (como el color rosa o el arco iris). En ellas predomina la publicidad (mucha de ella relacionada con el sexo). AmbienteG 
(www.ambienteg.com/, Facebook, Twitter), por ejemplo, combina en su primera página una guía gay de Manchester y la noticia de la penalización de la homosexualidad en Indonesia. Universo Gay (www.universogay.com, Facebook, Twitter y Youtube) es la más colorida: ofrece variedad de noticias (el orgullo LGTB en Serbia, por ejemplo), junto con publicidad, un chat gay y un servicio de citas en línea.

Cáscara Amarga (www.cascaraamarga.es, Facebook y Twitter) ofrece "periodismo y diversidad", centrándose en información cultural (obras de teatro relacionadas con lo LGTB, por ejemplo, o la relación que un candidato brasileño establece entre pederastia y homosexualidad). La publicidad es muy visible. Tiene pestañas sobre deportes, política LGTB, salud, sociedad, actualidad, etc.

Como vemos, las webs activistas son solo la punta de lanza del gran número de webs y otros recursos digitales existentes relacionados con cuestiones LGTB que se actualizan constantemente. De acuerdo con nuestros informantes, las más populares son Dos manzanas y Cáscara amarga. La página Dos manzanas fue creada en 2005 y es visitada por una media de 3200 personas al día. Ofrece noticias relacionadas con las personas LGTB e información general y permite que los usuarios hagan comentarios. Incluye algo de publicidad y participa en campañas activistas incorporando cuestionarios e información sobre campañas activistas.

Cáscara Amarga fue creada en 2010 para ofrecer información sobre la realidad LGTB. De acuerdo con sus datos, cuenta con 9.338 seguidores y también tiene un canal de televisión. Su estructura es como la de un periódico, con un director, editores, jefes de redacción, etc. Como en el caso anterior, incluye información sobre campañas y eventos activistas como el orgullo LGTB.

La prensa digital LGTB (como se autodefine Cáscara amarga) representa un modelo diferente de intervención en la sociedad: la combinación de noticias LGTB, noticias en general, información sobre reuniones LGTB y publicidad llena un vacío y se diferencia de las webs activistas. Su relación con las redes sociales es mucho más fluida que en el caso de las páginas activistas. Estas webs estables están orientadas a un público general que no necesariamente comparte una ideología política ni pertenece a un colectivo LGTB. Vinculan a personas LGTB con productos y espacios LGTB a través de la información y la publicidad, construyendo así flujos móviles de informaciones (más amplias que las 
proporcionadas por las webs activistas) basadas en el conocimiento, las prácticas y las experiencias compartidas.

\section{PROTESTA PÚBLICA Y PRÁCTICAS DIGITALES: DOS ESTUDIOS DE CASO EN BARCELONA Y EN MADRID}

Para analizar las prácticas digitales en relación con la esfera LGTB es pertinente averiguar qué elementos se ponen en funcionamiento para organizar una movilización. Aquí analizamos dos casos de protestas públicas: la concentración frente a la sede del Partido Popular (PP) en Madrid, organizada por COGAM (23 de marzo de 2011), y una besada masiva durante la visita del Papa a Barcelona (7 de noviembre de 2010).

El 7 de noviembre de 2010, el Papa Benedicto XVI visitó Barcelona y algunos grupos promovieron un kiss-in (besada masiva) frente a la catedral para protestar contra la postura homófoba de la Iglesia católica. Según las noticias publicadas, la protesta consistió en "un centenar de activistas homosexuales (que) critican con un 'acto de amor reivindicativo' la postura del Vaticano sobre la libertad sexual"18. El diario Público dijo que el "colectivo homosexual" organizó una besada masiva frente a la catedral, bajo el lema "Yo no te espero"19 ${ }^{19}$ como contraste con los seguidores del Papa, que sí esperaban ansiosamente su visita.

Vale la pena prestar atención a la utilización, en estas noticias, de las palabras "activista" y "colectivo". En particular, la idea de "colectivo

Figura 3

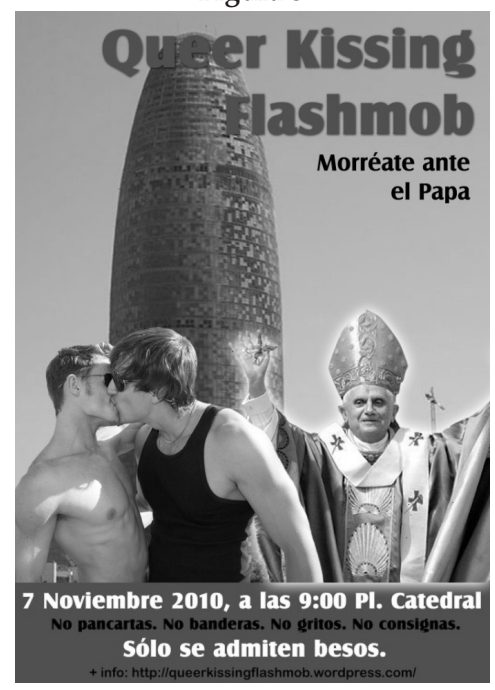


homosexual" nos remite a la visión de una comunidad estable y homogénea basada en afinidades - identitarias - que fue discutida en la primera parte de este artículo. Si nos fijamos en las imágenes de la protesta, veremos que los significantes tradicionales del activismo (pancartas, consignas, banderas) no son visibles durante la movilización. De hecho, si nos fijamos en el cartel creado para el evento, notaremos que expresamente dice: "No pancartas. No banderas. No gritos. No consignas. Sólo se admiten besos".

Los colectivos activistas, en cuanto tal, fueron invisibles en esta acción. Las fotos mostraron grupos de parejas (fundamentalmente de hombres) besándose. Por tanto, la etiqueta "activista" en las palabras de los medios se refiere más a su etimología (aquel/lla que actúa) y no a la pertenencia a un colectivo activista formal, información que los medios no tendrían pues nadie estaba sosteniendo una bandera de un colectivo. "Estar allí" en ese momento en particular y en protesta contra el Papa convirtió a esas personas en un "colectivo activista".

Este acto de protesta pública consistió en un flashmob, repertorio de protesta basado en una acción pública relámpago y multitudinaria, organizada desde la página Queer Kissing Flashmob (http://queerkissingflashmob.wordpress.com $)^{20}$. En esa página se daban instrucciones a los participantes y una vez terminada la acción, la página incluyó una extensa revisión de lo sucedido.

La preparación de esta acción fue controvertida: Queer Kissing Flashmob y su perfil abrieron una página en Facebook que reunió a 1.500 personas e invitó a 12.000 personas, pero fue cerrada por la censura de esta red social. Tras crear otro grupo que no fue censurado, 110 personas confirmaron su participación y, finalmente, 200 personas participaron efectivamente en la acción ${ }^{21}$.

Para la consecución de la movilización se activaron una serie de medios distintos (una página web, un blog, una cuenta de Facebook, Twitter y YouTube):

No nos sorprendería si Facebook prohíbe una vez más este evento, por lo que NECESITAMOS QUE CADA UNO DE VOSOTROS DIFUNDA ESTA INFORMACIÓN (por correo electrónico y todas las demás redes sociales) A CUANTAS PERSONAS PUEDA. Esta es la etapa final de nuestros esfuerzos: dar a conocer el evento tanto como sea posible 
garantizará nuestro éxito. Si de nuevo nos censuran en Facebook, por favor buscad las actualizaciones en nuestro BLOG.

Esperamos veros el 7 de noviembre en la Plaza de la Catedral de Barcelona a las 9 en punto de la mañana.

Besos Queer a todxs!

PD No hay que olvidar que se trata de una oportunidad ÚNICA. El Papa no ha visitado Barcelona en casi 20 años, y puede tardar otros 20 años en volver.

PD El 7 de noviembre, nuestro hashtag de Twitter será \#popemob ${ }^{22}$.

Sin embargo, no se encontraron referencias al activismo aunque la página incluye un agradecimiento especial a Facebook y a algunos blogueros. En la página, la gente invocada fue referida como "participantes", "pueblo" y "acción" (no activistas), y se prestó especial atención a la cobertura de los medios, tanto en España como a nivel internacional.

De acuerdo con la página web, la besada masiva fue destacada en las noticias tanto a nivel nacional como a nivel internacional: "Estamos contentos de saber que nuestros esfuerzos demostraron lo mucho que la sociedad española ha cambiado". La página web también mostró el siguiente anuncio:

Desde su nacimiento, las redes sociales y la web 2.0 jugaron un papel clave en el éxito de este esfuerzo de abajo a arriba. Creemos que estos modelos han demostrado ser eficaces y serán útiles para futuras protestas. Además, la censura repetida de Facebook (de las páginas del evento, otras páginas y numerosas cuentas de usuario) nos catapultó a la fama en los medios de comunicación.

La acción fue considerada como un "éxito personal" porque seis amigos fueron su origen, pero fue posible gracias a la colaboración de otras personas y de las redes sociales:

A nivel personal, el evento fue muy enriquecedor para nosotros: un pequeño grupo de amigos tuvo una idea simple y ha demostrado que podemos tener un impacto significativo para cambiar las cosas. Nunca antes habíamos tenido tanto éxito en nuestra lucha contra las posiciones de la Iglesia católica. Hemos logrado nuestros objetivos: lograr la visibilidad internacional y provocar un debate social $^{23}$.

El segundo caso de estudio elegido es una manifestación frente a la sede del conservador Partido Popular en Madrid. COGAM promovió esta acción colectiva a través de su página web. El objetivo era protes- 
tar contra el recurso del PP contra el matrimonio homosexual ante el Tribunal Constitucional (recurso que finalmente fue desestimado en 2013). La protesta tuvo lugar el 23 de marzo de 2011 y 200 personas participaron en ella. Después del evento, buscamos la protesta en Google y se realizó una búsqueda el 7 de abril de 2011 para averiguar el tipo de webs que habían informado sobre la protesta y analizar la relevancia de las redes de activistas. A través de esa búsqueda, podríamos tener más información sobre el tipo de sitios web que conforman la esfera LGTB utilizando el propio Internet como medio de comunicación.

Como era de esperar, la acción estaba referenciada en la página web de COGAM. A continuación, aparecía indexada en distintas páginas web (www.estanochegay.com; www.cristianosgays.com; noticias.universogay.com), fuentes de información para las personas LGTB. Después de estas tres webs, aparecían en la web de la FELGTB y en Dos manzanas, que es una de las webs más activas en proporcionar información actualizada sobre cuestiones LGTB en el mundo, y particularmente en España. Seguían distintas páginas dedicadas a la información. Por tanto, la acción fue más difundida a través de medios no activistas que de medios activistas (con excepción de la entidad convocante).

Hay que destacar que ambos eventos fueron posibles gracias a grupos de amigos que, en el caso del flashmob, claramente se identificaron como manifestantes informales y no institucionalizados. Los activistas digitales se consideran a sí mismos como diferentes de los militantes en colectivos activistas:

Más de doscientas personas asistieron al kiss-in, de pie a ambos lados de la plaza de la Catedral mientras el Papa la cruzaba. Estas personas estaban dispuestas a levantarse temprano un domingo por la mañana para prestar su apoyo en favor de los diversos tipos de amor que existen en el mundo. Nuestros esfuerzos fueron más significativos que todas las campañas de sensibilización patrocinadas institucionalmente ${ }^{24}$.

También vale la pena destacar que incluso en el caso de la movilización promovida por COGAM, la mayor parte de la información circuló a través de redes informales, lo cual nos lleva a pensar sobre el perfil del activismo LGTB en España en el marco de tendencias más amplias del activismo contemporáneo. 


\section{ACTIVISMOS FRAGMENTADOS Y COMUNIDADES MÓVILES: CONSIDERACIONES FINALES}

La construcción de una esfera LGTB combina elementos locales (colectivos activistas, locales de ocio), globales y digitales. Las prácticas y experiencias LGTB y el activismo se localizan y se arraigan en un espacio físico como muestra el énfasis de muchas webs activistas en la creación de grupos de trabajo y otros elementos presenciales (comisiones de trabajo).

La mayoría de los colectivos activistas combinan asesoramiento (ayuda, consultorías, asistencia) y activismo (campañas, organización de protestas, eventos del Orgullo). Anclados en lo local, el alcance de sus acciones de asesoramiento y acompañamiento llega sólo a un número limitado de personas; pero sus discursos y reivindicaciones han alcanzado un enorme impacto en la población y en la política española, produciendo grupos estables (COGAM, por ejemplo, tiene más de 25 años de existencia; la FELGTB fue creada en 1992). Son también un marco de referencia para la gestión de las identidades a través de sus consultorías y asesoramiento. Tanto el enaltecimiento de actividades comunes como el propio activismo juegan un papel importante para la construcción de la comunidad y de las identidades colectivas.

Las tecnologías digitales han promovido enormes cambios en las prácticas comunicativas y la sociabilidad: donde los públicos locales son limitados, los públicos digitales son, en teoría, ilimitados. Redes sociales como Facebook y Twitter, páginas web especializadas, blogs y teléfonos inteligentes proveen información y se utilizan como medios para la promoción de acciones de movilización como el kiss-in. Pero, como hemos visto, las redes sociales tradicionales (físicas) siguen siendo fundamentales en cómo los colectivos se piensan a sí mismos y se estructuran en torno a actividades presenciales. Esas redes también son fundamentales para el uso del ambiente como espacio de sociabilidad y de ocio y también lo son para la organización de las acciones de movilización: el uso de las tecnologías para la protesta se basa en gran medida en el recurso a redes preexistentes de conexiones sociales (amigos, colegas y otros activistas). En este sentido, las prácticas de sociabilidad auspiciadas por COGAM y otros colectivos (actividades de cine y de teatro, excursiones, clases, etc.) ayudan a definir y a cohesionar los grupos que pasarán a la acción llegado el momento, utilizando tecnologías no digitales y digitales. Las redes físicas de activistas, tête-à-tête, 
coexisten con una esfera digital en la que, además de páginas activistas, existen otras páginas especializadas y páginas orientadas a fines específicos (como, por ejemplo, la página para el flashmob). La combinación de redes físicas, digitales y otros medios de información conforma hoy lo que Warner llamó "contra-públicos":

Por definición, los contra-públicos están formados por su conflicto con las normas y los contextos de su entorno cultural, y este contexto de dominación inevitablemente conlleva distorsión. Es decir, los públicos masivos y los contra-públicos son formas de lo público dañadas, de igual modo que en esta cultura, el género y la sexualidad son formas dañadas de privacidad (Warner, 2002:63) ${ }^{25}$.

Pero el activismo no solo da consistencia a los contra-públicos y sostiene la comunidad, sino que, como Taylor apuntó (Taylor et al., 2002), también la fragmenta. Muchos de los que utilizan medios digitales para organizar protestas sociales fuera de los círculos del activismo reglado y formal no se consideran activistas pero comparten una ideología, una opinión, y la necesidad de organizar la protesta social informal. Como Warner mostró, la esfera pública no es esa utopía liberal democrática donde el debate continuo garantiza la igualdad. El público no es una entidad homogénea, sino que se compone de múltiples contra-públicos reflexivos. Los públicos y contra-públicos están en constante flujo y definición: en esa definición, intervienen todos y cada uno de los elementos considerados en este trabajo (digitales y no digitales, activistas y no activistas, relacionados con la ideología y con el tiempo libre, etc.). Comparten un objetivo: la igualdad de derechos y la lucha contra la discriminación.

Esto tiene algunas consecuencias: la primera se refiere a cómo podemos (o debemos) concebir hoy una comunidad que ya no puede ser pensada como un todo cerrado, sino abierto, permeable y flexible, tal y como Steward y Wolf prefiguraron, y se discutió en la primera parte de este artículo. Las comunidades son ahora configuraciones móviles que pueden estar tan fracturadas y ser tan versátiles como el propio activismo (Tejerina, 2010:273). Las prácticas y potencialidades digitales han incorporado una mayor complejidad y heterogeneidad a nuestra comprensión de las comunidades y sus límites.

Otra cuestión que se deriva de nuestro análisis se refiere a la definición y al alcance del activismo: debemos superar las falsas dicotomías que oponen el activismo formal al informal y el activismo de base al digi- 
tal $^{26}$. Como hemos visto, acciones, flujos y estrategias digitales y no digitales son necesarias para la existencia de ambas, y están profundamente entrelazadas en la construcción de la esfera LGTB.

La actividad digital de los colectivos activistas LGTB es a veces limitada y, como vimos, sus páginas web no permiten la interactividad con los usuarios y son principalmente informativas. No obstante, su potencial para llegar a enormes cantidades de personas y organizar eventos es enorme, como prueba el gran número de participantes en la manifestación del Orgullo de Madrid (más de un millón de personas). Situados en el centro de la esfera pública LGTB y como guardianes de las reivindicaciones y el discurso LGTB, los colectivos activistas están en el centro de configuraciones o de círculos concéntricos, donde las redes de activistas y sus conexiones - digitales y no digitales - sirven como conductores del discurso activista que potencia el sentido de pertenencia a una comunidad. El activismo está en el centro de redes sociales más amplias que usan tecnologías digitales y no digitales. Como la mayoría de las secciones de los colectivos (jóvenes, mayores, excursiones) requieren la comunicación cara a cara, su efecto socializador es una potente estrategia para la construcción de la integración (entre ellos) y de la diferencia (con los otros) (Tejerina, 2010) y, por lo tanto, para profundizar la sensación de pertenencia que subyace a la noción de comunidad.

Esa pertenencia favorece la existencia de otras expresiones como las webs informativas, los sitios de citas digitales, la publicidad especializada (después de la constitución de un claro nicho de mercado), el ambiente, etc., cuya relación con el activismo a veces es problemática debido a sus diferentes objetivos y expresiones. Hoy en día, todas las expresiones LGTB están estrechamente relacionadas con el uso intensivo de las tecnologías digitales tanto por públicos como por el contrapúblicos. Lo digital ya no existe separadamente, sino en continua conexión con lo no digital, y los flujos de información, asesoramiento, acciones activistas, reivindicaciones y demandas serían ahora imposibles sin la complicidad entre ambos.

En consecuencia, el espacio físico compartido (para el activismo o el ocio), los discursos (activistas) disponibles, los recursos informativos (sobre todo digitales), la comunicación (en blogs, webs estables y periódicos digitales) y la protesta social son elementos que forman unos (contra) públicos que se imaginan a sí mismos como una comunidad - 
en el sentido que Anderson (1991) ${ }^{27}$ dio a las "comunidades imaginadas" - que conforma la esfera LGTB con deseos, experiencias, prácticas, emociones y conocimiento compartidos.

(Recebido para publicação em abril de 2013) (Reapresentado em outubro de 2014 e em fevereiro de 2015) (Aprovado para publicação em fevereiro de 2016) 


\section{Begonya Enguix Grau}

\section{NOTAS}

1. COGAM (Colectivo de lesbianas, gays, transexuales y bisexuales de Madrid); FELGTB (Federación Estatal de LGTB); Cogailes (Coordinadora Gai-Lesbiana); FAGC (Front d'Alliberament Gai de Catalunya).

2. Esta lista no pretende ser exhaustiva, sino informativa. Cáscara amarga (www.cascaraamarga.es), Universo Gay (www.universogay.com/), En sentido contrario (www.ensentidocontrario.com), Ocio gay (www.ocioxocio.com/category/ociogay/), Dos manzanas (www.dosmanzanas.com/), y AmbienteG (www.ambienteg.com).

3. Una aproximación a la conceptualización de las comunidades se encuentra en Martinez Veiga (2012).

4. En el original: "(they are) social aggregations that emerge from the net when enough people carry on... public discussions long enough, with sufficient human feeling, to form webs of personal relationships in cyberspace" (Rheingold, 1993:3). Esta y todas las traducciones, cuyas transcripciones se reproducen en las siguientes notas, son de la autora del presente artículo.

5. En el original: "a community is not fixed in form or function, it is a mixed bag of possible options whose meanings and concreteness are always being negotiated by individuals, in the context of changing external constraints. This is true whether group members interact electronically, via face-to-face communication, or both" (en Kozinets 2010:9).

6. Véanse, por ejemplo, Chauncey (1994), Davis (1995), Knopp (1995), Hubbard (2004) y Higgs (1999).

7. En el original: “the conversion of place into space intervenes to produce an 'authenticity' that enables political claims to be made and contested. Space is not just a passive backdrop to human behaviour but is constantly produced and remade as groups struggle for power (or against powerlessness) to convert their 'reality' into public knowledge, in which a visible presence can be recognised" (Skeggs et al., 2004:1839).

8. En el barrio de Chueca reside mucha población LGTB que convive con todo tipo de negocios dedicados a esa población (desde librerías a discotecas).

9. Estas posiciones son en buena parte idealizaciones, pues en colectivos asimilacionistas también encontramos posturas queer.

10. Coordinadora activa en el momento inicial de la investigación y de escritura del artículo, pero inactiva a partir de 2015.

11. Todas las páginas web fueron consultadas el 1, 2 y 3 de octubre de 2014.

12. Se trata de fans del festival de música Eurovisión.

13. Todos los datos fueron extraídos el 30 de septiembre 2014.

14. Esta página fue consultada el 27 de febrero de 2013.

15. Entrevisté a Eugeni Rodríguez el 6 de mayo de 2014.

16. Como ejemplo, podemos comparar las webs del Orgullo LGTB: la del Barcelona Pride, organizado por una asociación empresarial y muy mercantilizado, incluye a los patrocinadores del evento. También lo hace la web de actividades en torno a la manifestación estatal del Orgullo LGTB, a cargo de AEGAL, asociación empresarial LGTB de Madrid (ver http://www.pridebarcelona.org/ y http://www.madridorgullo.com/es/). Sin embargo, la web oficial del Orgullo de Madrid, controlada por acti- 
vistas y centrada en la manifestación, no incluye ni publicidad ni el patrocinio (http:/ / orgullolgtb.org/en, todas las páginas consultadas el 19 de marzo de 2013).

17. Todas las páginas fueron recuperadas el 1 de octubre de 2014, excepto cuando se indica lo contrario.

18. Disponible en http://www.elperiodico.com/es/noticias/sociedad/20101107/ beso-colectivo-gay-protesta-por-visita/582877.shtml (consultada el 16 de octubre de 2011).

19. Disponible en http://www.publico.es/espana/345502/barcelona-acoge-la-visita-con-frialdad (consultada el 16 de octubre de 2011).

20. Consultada el 16 de octubre de 2011 y el 19 de marzo de 2013.

21. En http://www.redescristianas.net/2010/10/25/besos-gais-contra-la-visita-del-papa-a-bcn/, consultada el 16 de octubre de 2011.

22. Extraído de http:/ / queerkissingflashmob.wordpress.com el 2 de octubre de 2014. Énfasis original.

23. Todas las citas proceden de http:/ / queerkissingflashmob.wordpress.com y fueron extraídas el 19 de marzo de 2013.

24. En http:/ / queerkissingflashmob.wordpress.com/ page (consultada el 19 de marzo de 2013).

25. En el original: "Counterpublics are, by definition, formed by their conflict with the norms and contexts of their cultural environment, and this context of domination inevitably entails distortion. Mass publics and counterpublics, in other words, are both damaged forms of publicness, just as gender and sexuality are, in this culture, damaged forms of privacy" (Warner, 2002:63).

26. El activismo de base es llamado, en inglés, grassroots. En 2002 Jerome Armstrong acuñó el término netroots para referirse al activismo político organizado mediante blogs y otros medios digitales (incluyendo wikis y redes) (en http://johnpostill.wordpress.com/, consultada el 18 de octubre de 2011).

27. Para Anderson, las comunidades imaginadas lo son porque sus miembros nunca conocerán a sus compañeros, ni los encontrarán, ni incluso oirán hablar de ellos, "aunque en sus mentes vive la imagen de su comunión"... De hecho, todas las comunidades mayores de los pueblos primarios con contacto cara-a-cara (e incluso éstas) son comunidades imaginadas (1991:49). 


\section{Begonya Enguix Grau}

\section{REFERENCIAS}

ALDRICH, Robert. (2004), "Homosexuality and the City: An Historical Overview". Urban Studies, vol. 41, no 9, pp. 1719-1737.

ANDERSON, Benedict. (1991), Imagined Communities: Reflections on the Origin and Spread of Nationalism. London, Verso (rev. ed.).

ARMSTRONG, Elizabeth A. (2002), Forging Gay Identities. Organizing Sexuality in San Francisco, 1950-1994. Chicago, The University of Chicago Press.

CHAUNCEY, George. (1994), Gay New York: Gender, Urban Cultures and the Making of the Gay Male World 1890-1940. New York, Basic Books.

CRUCES, Francisco. (1998), “Las Transformaciones de lo Público. Imágenes de Protesta en la Ciudad de México". Perfiles Latinoamericanos, no 12, pp. 227-256.

DAVIS, Tim. (1995), "The diversity of Queer Politics and the Redefinition of Sexual Identity and Community in Urban Spaces", in D. Bell; G. Valentine (eds.), Mapping Desires. Geographies of Sexualities. New York, Routledge, pp. 284-303.

DE CERTEAU, Michel. (1984), The Practice of Everyday Life. Berkeley, University of California Press.

FINQUELIEVICH, Susana (ed.). (2000), ¡Ciudadanos, a la Red!: Los Vínculos Sociales en el Ciberespacio. Buenos Aires, Ciccus.

FORSYTH, Ann. (2001), "Nonconformist Populations and Planning Sexuality and Space: Nonconformist Populations and Planning Practice". Journal of Planning Literature, no 15, pp. 339-358.

FOUCAULT, Michel. (1984), Historia de la Sexualidad (I). La Voluntad de Saber. Madrid, Siglo XXI.

HABERMAS, J. (1989), The Structural Transformation of the Public Sphere: An Inquiry into a category of Bourgeois Society. Cambridge, Polity.

HANDS, Joss. (2011), @ is for activism. Dissent, Resistance and Rebellion in a Digital Culture. New York, Pluto Press.

HIGGS, David (ed.). (1999), Queer Sites. Gay Urban Histories since 1600. London, Routledge.

HUBBARD, Phil. (2001), "Sex Zones: Intimacy, Citizenship and Public Space". Sexualities, vol. 4, no 1, pp. 51-71.

(2012), Cities and Sexualities. New York, Routledge.

KNOPP, Lawrence. (1995), "Sexuality and Urban Space: A Framework for Analysis", in D. Bell; G. Valentine (eds.), Mapping Desires. Geographies of Sexualities. New York, Routledge, pp. 149- 164.

KOZINETS, Robert V. (2010), Netnography. Doing Ethnographic Research Digital. London, Sage.

MARTÍNEZ VEIGA, Ubaldo. (2012), Historia de la Antropología. Formaciones Socioeconómicas y Praxis Antropológicas, Teorías e Ideologías. Madrid, UNED. 
MEYROWITZ, Joshua. (2004), Glocality. New Senses of Place and Identity in the Global Village. Disponible en http://21st.century.phil-inst.hu/Passagen_eng14_Meyrowitz.pdf. Consultada el 10 de enero 2013.

REDFIELD, Robert. (1956), Peasant Society and Culture. Chicago, University of Chicago.

RHEINGOLD, Howard. (1993), The Virtual Community. Homesteading in the Electronic Frontier. Reading (MA), Addison-Wesley Publishing Company.

SKEGGS, Beverley et al. (2004), "Queer as Folk: Producing the Real of urban Space". Urban Studies, vol. 41, pp. 1839-1856.

SOULES, Marshall. (2008), Jürgen Habermas and the Public Sphere. Disponible en http://records.viu.ca/ soules/media301/habermas.htm. Consultado el 18 de octubre de 2011.

STEWARD, Julian, et al. (1956), The People of Puerto Rico. Urbana, University of Illinois Press.

TAYLOR, Verta; KAMINSKY, Elizabeth; DUGAN, Kimberly. (2002), "From the Bowery to the Castro: Communities, Identities and Movements", in D. Richardson; S. Seidman (eds.), Handbook of Lesbian and Gay Studies. London, Sage, pp. 99-114.

TEJERINA, Benjamín. (2010), La Sociedad Imaginada. Madrid, Trotta.

VAN INGEN, Cathy. (2003), "Geographies of Gender, Sexuality and Race: Reframing the Focus of Space in Sport Sociology". International Review for the Sociology of Sport, vol. 38, pp. 201-216.

WARNER, Michael. (2002). Publics and Counterpublics. New York, Zone Books.

WOLF, Eric. (1971), Los Campesinos. Madrid, Labor. 


\section{RESUMO}

Ativismo e Práticas Digitais na Construção de uma Esfera LGBT na Espanha

A partir de um questionamento antropológico sobre comunidades e a construção de uma esfera pública, este artigo aborda as estratégias digitais que servem para compor o perfil do que poderia ser denominado como esfera LGBT espanhola. Consideramos o ativismo LGBT como o principal produtor de discurso social legitimado e, através dele, analisamos os websites de sete coletivos LGBT e outros recursos digitais, no intuito de compreendermos a articulação de práticas digitais e não digitais, as quais estão baseadas em um entendimento compartilhado que evoca as identidades coletivas e retroalimenta o ativismo. Ao combinarmos o trabalho de campo antropológico sobre ativismo e etnografias digitais de ambientes virtuais, acreditamos que, em que pese o uso intensivo de recursos digitais, a esfera LGBT continua dependendo em larga medida das redes sociais tradicionais. Consequentemente, questionamos a utilidade da conceituação das relações digitais e não digitais como campos separados e distintos e discutimos essa imbricação como uma característica do ativismo contemporâneo.

Palavras-chave: práticas digitais; redes sociais; ativismo LGBT; mobilização; comunidades

\section{ABSTRACT \\ Activism and Digital Practices in the Construction of an LGTB Sphere in Spain}

Based on the anthropological interrogation into communities and the construction of a public sphere, this article approaches the digital strategies used to shape what may be referred to as the Spanish LGTB sphere. I consider LGTB activism as the main producer of legitimized social discourse, and have therefore analyzed the websites of seven LGTB collectives and other digital resources in order to examine the articulation of digital and non-digital practices, based on a shared knowledge evoking collective identities and feeding activism. In combining anthropological fieldwork on activism and digital ethnographies for virtual environments, I suggest that despite their intensive use of digital resources, the LGTB sphere still largely depends on traditional social networks. As a consequence, the article questions the usefulness of conceiving of digital and non-digital relations as separate and distinct and discusses this embeddedness as a main feature of contemporary activism.

Key words: digital practices; social networks; LGTB activism; mobilization; communities 
RÉSUMÉ

Activisme et Pratiques Numériques dans la Construction d'une Sphère LGBT en Espagne

À partir d'une réflexion anthropologique sur les communautés et la construction d'une sphère publique, cet article aborde les stratégies numériques servant à donner corps à ce que l'on pourrait appeler la sphère LGBT espagnole. Nous considérons l'activisme LGBT comme le principal producteur de discours social légitime et c'est pour cette raison que nous avons analysé les pages web de sept collectifs LGBT, ainsi que d'autres ressources en ligne, pour analyser l'articulation de pratiques numériques et non numériques qui se basent sur une connaissance partagée évoquant les identités collectives et alimentant l'activisme. En combinant un travail anthropologique de terrain et des ethnographies numériques d'environnements virtuels, nous considérons qu'en dépit de l'usage intensif des ressources en ligne, la sphère LGBT continue de dépendre des réseaux sociaux traditionnels. En conséquence, nous remettons en question l'utilité de la conceptualisation des relations numériques et non numériques comme étant séparées et distinctes et nous. Enfin, nous discutons cette imbrication comme une caractéristique de l'activisme contemporain.

Mots-clés: pratiques numériques; réseaux sociaux; activisme LGBT; mobilisation; communautés

\section{RESUMEN \\ Activismo y Prácticas Digitales en la Construcción de una Esfera LGTB en España}

A partir de una interrogación antropológica sobre las "comunidades" y la construcción de una "esfera pública", este artículo aborda las estrategias digitales que sirven para dar forma a lo que podría denominarse como la esfera LGTB española. Consideramos el activismo LGTB como el principal productor de discurso social legitimado y por ello hemos analizado las páginas web de siete colectivos LGTB y otros recursos digitales para examinar la articulación de prácticas digitales y no digitales, que se basan en un conocimiento compartido que evoca las identidades colectivas y retroalimenta el activismo. Tras combinar trabajo de campo antropológico sobre el activismo y etnografías digitales de entornos virtuales, consideramos que a pesar del uso intensivo de los recursos digitales, la esfera LGTB sigue dependiendo en gran medida de las redes sociales tradicionales. Como consecuencia, ponemos en duda la utilidad de la conceptualización de las relaciones digitales y no digitales como separadas y distintas y discutimos esa imbricación como una característica del activismo contemporáneo.

Palabras clave: prácticas digitales; redes sociales; activismo LGTB; movilización; comunidades 